These findings suggest that the apparent acute onset of IDD in women aged over 18 years is often illusory. Women aged over 19 years with IDD $^{18}$ and even younger patients ${ }^{19}$ may have a prolonged symptomatic illness before diagnosis, while Fajans et $a l^{20}$ described chemical diabetes and abnormal insulin responses existing more than eight years before the development of overt IDD. Similarly, the pancreatic islet cell antibody has been described in patients, with other organ-specific autoimmune diseases, many years before the development of clinical IDD. ${ }^{21}$ A study of haemoglobin $A_{1}$ concentrations in newly diagnosed juvenile-onset IDDs has confirmed that the prediagnosis period of hyperglycaemia often extends far beyond the duration of diabetic symptoms. ${ }^{22}$

This concept of an extended period of presymptomatic carbohydrate intolerance in a patient with IDD is compatible with that of a non-specific viral illness precipitating the onset of symptoms of clinical diabetes and hence with a seasonally variable incidence. Most patients included in the present study, however, developed clinical diabetes over the age of 20 years and all were parous women. It has recently been shown that such patients with IDD fail to exhibit a seasonal variation in incidence. ${ }^{18}{ }^{23}$

Two alternative hypotheses may therefore be proposed to explain the present findings. One supposes that more than one category of juvenile-onset diabetes exists, some cases being associated with a lengthy prediabetic phase and others being of genuine acute onset perhaps resulting from a viral infection. Alternatively, a prediabetic metabolic abnormality may be a general characteristic of IDD, acute viral illness merely acting as a trigger to precipitate clinical presentation of the underlying disease in some cases. At present it is not possible to choose between these hypotheses.
We should like to thank Mrs Sarah Dickson for her secretarial help and Professor J W Farquhar for his advice.

\section{References}

1 Cudworth, A G, British fournal of Hospital Medicine, 1976, 16, 207.

2 Irvine, W J, Lancet, 1977, 1, 638 .

${ }^{3}$ Ganda, O P, and Soeldner, S S, Archives of Internal Medicine, 1977, 137, 461.

4 Gamble, D R, and Taylor, K W, British Medical fournal, 1969, 3, 631.

5 Bloom, A, Hayes, T M, and Gamble, D R, British Medical Fournal, 1975, 3, 580 .

${ }^{6}$ Gamble, D R, et al, British Medical fournal, 1969, 3, 677.

${ }^{7}$ Lily, F, and Pincus, T, Advances in Cancer Research, 1973, 17, 231.

${ }^{8}$ Pedersen, J, The Pregnant Diabetic and her Newborn, p 48. Copenhagen, Munksgaard, 1977.

${ }^{9}$ Malins, J M, and Fitzgerald, M G, Diabetes, 1965, 14, 175.

10 Gairdner, D, and Pearson, J, Archives of Disease in Childhood, 1971, 46, 783.

11 Walker, C H M, Scottish Neonatal Annual Statistics, University of Dundee Medical School, 1977.

12 Malins, J M, Clinical Diabetes Mellitus, p 29. London, Eyre and Spottiswoode, 1968.

13 Skipper, E, Quarterly fournal of Medicine, 1933, 2, 353.

${ }_{14}$ Miller, H C, New England fournal of Medicine, 1945, 233, 276.

${ }^{15}$ Kriss, J P, and Futcher, P H, fournal of Clinical Endocrinology, 1948, 8, 380.

16 Hagbard, L, Acta Obstetricia et Gynecologica Scandinavica, 1958, 37, 497.

17 Tattersall, R B, Quarterly fournal of Medicine, 1974, 170, 339.

18 Gray, R S, Duncan, L J P, and Clarke, B F. In preparation.

19 Hamilton, D V, Mundia, S S, and Lister, R J, British Medical fournal, 1978, 2, 211.

${ }^{20}$ Fajans, S S, Archives of Internal Medicine, 1976, 136, 194.

${ }^{21}$ Irvine, W J, et al, Lancet, 1977, 1, 1025.

${ }^{22}$ Spack, N, and Sendzischew, S, Diabetes, 1978, 27, suppl No 2, p 464.

${ }^{23}$ Christau, B, et al, Diabetologia, 1978, 15, 224.

(Accepted 2 April 1979)

\title{
Evidential value of the hospital record in clinical decision making
}

\author{
W I CARD, W SIRCUS, A N SMITH
}

British Medical fournal, 1979, 1, 1305-1308

\section{Summary and conclusions}

A simulated retrospective exercise in the diagnosis and management of 53 readmissions to a gastrointestinal unit was undertaken by two consultants. Diagnosis of the illness at readmission was made on evidence sought from a referee, who also supplied, on request, items of relevant evidence from the past medical record. Patient management was agreed from these sources. For each item of evidence the evidential weight, the irrecoverability, and the expected benefit accruing to the patient of its availability was calculated.

\footnotetext{
Diagnostic Methodology Research Unit, Southern General Hospital, Glasgow G51 4TF

W I CARD, MD, FRCP, emeritus professor, University of Glasgow

Gastrointestinal Unit, Western General Hospital and University of Edinburgh, Edinburgh EH4 2XU

W SIRCUS, PHD, FRCP, reader in medicine, University of Edinburgh A N SMITH, MD, FRCS ED, reader in clinical surgery, University of Edinburgh
}

It was concluded that the evidence worth recording in the event of subsequent hospital admission could be largely specified for each diagnosis and each operation. It would be brief and could be numerically coded.

\section{Introduction}

In decision theory the medical record constitutes written information about the illness of a patient that may be relevant to current and future decisions. Such decisions may be of various kinds, and could be medicolegal or administrative or related to medical research, though more often and most important they are related to the current care of the patient and future care should he be readmitted.

Much interest had been aroused by the work of Weed, ${ }^{1}$ who suggested a method of presenting clinical data that he called the problem-oriented record (POR). This method must be distinguished from the conventional one of recording data, which has been termed the source-oriented record (SOR). ${ }^{2}$ The POR has received much favourable and some critical comment. We are concerned here not with the merits or demerits of the two systems but with the problem of determining what information should be included in the case record on the chance that it may influence clinical decisions in the future. 


\section{Background}

Application of the mathematics of probability and decision theory permits reconsideration of clinical medicine that can then be expressed in terms of a formal structure. ${ }^{4}$ In terms of this structure the clinical activity of a doctor may be described as a sequence of decisions (fig 1).

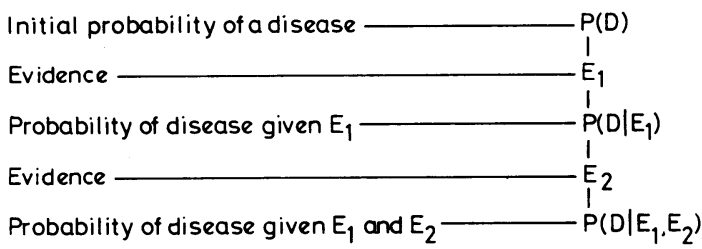

FIG 1-Sequential collection of evidence used in decision making.

Initially each doctor seeks further evidence about an illness by questioning the patient, examining him, or asking for laboratory or radiological investigations, and each decision is influenced by the evidence already gained. Finally, a decision is made on treatment. Some of the evidence the doctor needs may be contained in the record of a past illness.

Let us suppose that a patient is admitted with disease $\mathrm{A}$ and is treated and discharged (fig 2). A record of his illness is made and stored. He is subsequently readmitted with disease $B$. To diagnose disease $B$ the doctor will obtain most of the evidence he needs direct from the patient, but part of the evidence may be contained in the record of disease A. The problem then becomes: What facts about disease $A$ should be recorded and stored that will have value should the patient be readmitted with disease $B$ ?

Any such recorded fact has a certain average evidential value if it increases the accuracy of a diagnosis and thus has a favourable effect on the ultimate condition of the patient. In a completely formal system this improvement in health would be set against the cost of recording and storing the evidence. It could thus be decided whether recording this particular piece of evidence was "worth while." While such a completely formal system as suggested by Good and Card ${ }^{5}$ is not yet feasible, certain criteria may be identified that could influence the decision whether to record a clinical fact or not.

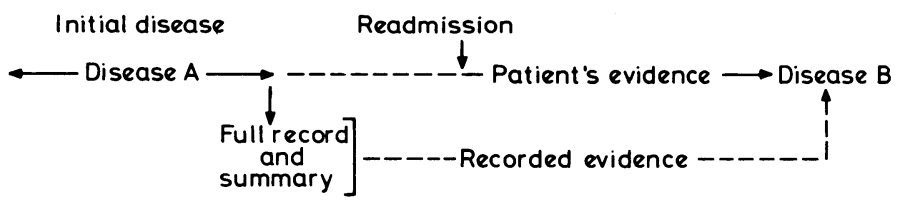

FIG 2-Flowpath in diagnosis after readmission.

\section{EVIDENTIAL VALUE OF CLINICAL FACT IN RELATION TO DISEASE B}

Certain facts may carry great evidential value-for example, that at a previous operation an enteroanastomosis has been performed. Other facts may be trivial and have little relevance to any disease. A particular fact may, of course, be relevant to more than one disease.

\section{PROBABILITY OR CHANCE THAT DISEASE B WILL OCCUR}

Disease B might be a recurrence of the same disease-for example, proctocolitis, when the chance of it occurring might be quite highor a complication of disease $\mathrm{A}$, such as anaemia after gastrectomy. On the other hand, disease $B$ might have no connection with disease $A$, and the chance of it occurring would then be quite unaltered by the knowledge that disease A has occurred.

Mathematically, the evidential value of the clinical fact in relation to disease $\mathbf{B}$ and the probability or chance that disease $\mathbf{B}$ will occur are combined by multiplying the evidential value by the probability or chance that it will be used. This combination is the evidential value that we may expect on the average.

\section{RECOVERABILITY OF EVIDENCE}

Recoverability refers here not to the physical recovery of a case record but to how easily information that should be in the record can be obtained from the patient on readmission. The patient will not normally know, for instance, what type of anastomosis has been performed at an operation for peptic ulcer. Such information cannot easily be recovered and may sometimes be obtained only by complex radiological examination or even laparotomy. On the other hand, an item such as the patient's blood group is readily obtainable on readmission and may thus be said to be easily recoverable. The evidence most important to record, other things being equal, is that which is not easily recoverable.

\section{BENEFIT OF TREATING DISEASE B SHOULD IT OCCUR}

Medicine is ultimately concerned with the benefit obtained by treating the patient. Evidence may be recorded that is of considerable evidential value for diagnosis and prognosis; the probability of a disease recurring may be high, as in malignancy; and the evidence may not easily be recoverable on readmission. In other words, all three criteria strongly imply that the evidence is of great value. But if little or no benefit is expected from treating the disease the evidence becomes almost worthless.

\section{Methods}

Records of patients with more than one hospital admission to a gastrointestinal unit were chosen at random from a sequential series of 350 case notes, beginning at least 10 years before to eliminate memory bias. For each patient a comprehensive discharge summary for transmission to the general practitioner had been prepared. This provided details of the initial illness, disease $A$, and included relevant laboratory, radiological, pathological, and operative data. The discharge summaries had been prepared by a senior registrar and vetted by a consultant. This policy has been used by the gastrointestinal unit in Edinburgh throughout its 27 years of existence.

For the present study a consultant colleague made out a very brief synopsis of the mode of presentation and relevant signs of the second illness, disease $\mathrm{B}$, including a diagnosis such as might have been provided by the general practitioner at the time of this referral; but no other information was given other than the patient's age, sex, initials, and hospital code number.

\section{SIMULATION STUDY}

The final methodology was established as follows after a preliminary pilot study.

A simulation exercise of the diagnosis and management of disease B was conducted on the basis of the brief information given for the second referral. This was undertaken without knowledge of the patient's identity by a consultant physician and consultant surgeon (WS and ANS) working in the unit. Another observer (WIC) held the case notes and discharge summaries and refereed on the presence or absence in them of the further information needed to reach a diagnosis and decide on management. The pattern of the exercise was as follows.

The two clinicians were asked by the referee what decisions were required to reach a correct diagnosis of disease $B$ and give adequate treatment. Each entered on a form the details of any evidence that could be obtained from the record of disease $A$ and whether this information was irrecoverable and the expected benefit, less cost, of the treatment that the clinician ultimately advised. Any such information used from the record of disease A was graded 0-5 in respect of evidential value, degree of irrecoverability, and expected benefit less cost. The exercise suggested that a substantial amount of the required historical evidence would have been obtainable from the patient on readmission. "Irrecoverability" was used instead of "recoverability" so that this scale moved in the same direction as the scales for the other variables. A completely recoverable piece of evidence-for example, blood group-would be scaled 0 . The benefit to be gained from treating disease $B$ is really expected benefit, since any treatment has only a certain chance of success, and the measurement of the chance-the probability-must be combined with the benefit that may occur. To be completely formal "cost" must be subtracted from the expected benefit, and we attempted to do this. Cost is a complex concept that includes monetary cost and factors such as danger, pain, anxiety, etc. This estimate of expected benefit less cost is necessarily extremely crude.

The probability or chance that disease B will occur is not mentioned above. It is not possible, with this retrospective method, to get any estimate of this probability, though it would be possible to get rough estimates from an analysis of hospital admissions. 


\section{Results}

The records of 53 patients with multiple admissions were studied. In two cases the record summary was deemed inadequate. In nine the record of disease $\mathrm{A}$ was irrelevant to the diagnosis and management of disease $B$ and was ignored. In the remaining 42 patients the past record was examined as requested by the consultant and each piece of evidence scored as described below.

It should be noted that the year of admission of most patients whose case notes were examined predated the use of fibreoptic endoscopy; the evidential value of this procedure would probably be higher.

It was thought useful to construct a total score for each piece of evidence. Two difficulties were encountered. Firstly, the scales of the different criteria should be compatible so that such scores may be properly combined. Secondly, some mathematical function must be devised to combine them. Adjustment of the scales of the separate variables was not attempted, though it was felt that the scaling of "cost" was much too high, and this variable was ultimately ignored. In devising the function one condition had to be met: that if any of the variables was zero the total score had to be zero. For example, however powerful a piece of evidence might be, if it is completely recoverable-that is, if its irrecoverability is zero-then the evidence need not be recorded since its total value is zero. This condition would be met by a multiplicative but not an additive function. We therefore calculated a total score for each piece of evidence by multiplying together the evidential value, the degree of irrecoverability, and the expected benefit (fig 3 ). The table gives the total scores.

\section{Previous admission:}

Readmission:

Evidence sought from past record: malignant gastric ulcer partial gastrectomy

general physical deterioration. ? epigastric mass pathology of lymph nodes?

\begin{tabular}{lc} 
Pathology report: malignant infiltration present \\
\hline Evidential value & 5 \\
Irrecoverability of evidence & 5 \\
Expected benefit & $\frac{1}{25}$ \\
& Total score
\end{tabular}

FIG 3-Example of process of scoring evidence (multiplicative function). Value of evidence was very high; evidence was not otherwise recoverable, but expected benefit was very small.

The scores ranged from 0 to 155 with a median of 24 . When a frequency distribution of the scores was plotted it was seen to be highly skewed, suggesting a log-normal distribution. When the logarithms of the scores were calculated and a frequency distribution plotted it approximated to the normal distribution. On our data alone it does not seem possible to decide between different distributions and therefore between the different functions from which they are derived. But we believe that the raw product of the scores unduly magnifies the differences between scores from different sources of evidence and that therefore the logarithm of the raw product may be a fairer measure. This slightly alters the ordering. The table shows that only five

Distribution of scores for each piece of evidence

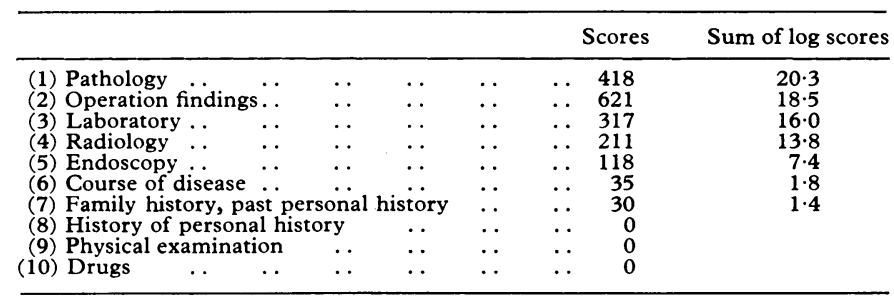

sources of evidence proved to be important-namely, pathology report, operation findings, laboratory findings, radiology, and endoscopy. The remainder could be ignored with little loss. Our comparison cannot go much further than putting all these sources of evidence in some kind of ranking order.

The information required from the past record was also sought in the summary and a comparison of the adequacy of the information from each source made. The information needed for managing the second or subsequent hospital admission, disease B, was present in the summaries of 39 of the 42 patients.

\section{Discussion}

We must emphasise that these conclusions may be true only of gastroenterology, and indeed may be true only of the particular unit that includes both medical and surgical patients under joint management by physicians and surgeons in the same ward. For instance, the record of physical signs in our patients was never requested, since in gastroenterology it mostly appears to be of little value. In neurology, however, it might be of great importance. Further studies in other specialties would be highly desirable. It would also be useful to have tested records of patients who had originally been admitted to the gastrointestinal unit but were subsequently readmitted to another ward for a non-gastroenterological complaint.

In this study records were analysed solely for their value to the patient should he be readmitted. Many feel that they are also valuable for clinical research. To be of real use, however, clinical research should probably be done prospectively with a clear objective in mind and an appropriate form on which to collect the necessary data. This was the view of Platt, ${ }^{6}$ who said that "more often there is little to be learnt which is not already known unless the records have been kept with an end in view, as part of a planned experiment. . . . Neither clinical record nor logic by themselves can replace the experimental method in clinical investigation as Harvey knew a long time ago." Doll? expressed similar views.

The objection to recording all information about the patient on the chance that something will later prove useful is that the cost of recording, storing, and subsequently decoding this information is not justified by any benefit that accrues. Apart from information that might help the individual patient if readmitted, any additional information can be recorded only as having some potential value to medical science. While in a fully fledged research project such information will have been carefully selected and a special form designed, it seems perfectly reasonable for, say, a consultant who suspects that there is some relation between halothane anaesthesia and subsequent jaundice to arrange for details of the anaesthetic to be recorded. But this then becomes research in however elementary a form. We therefore contend that, in general, the aims of clinical medical research and the needs of the individual patient are quite different, and these objectives should be clearly separated. In discussions on record keeping nothing has caused greater confusion than the assumption that these two largely incompatible aims can be realised in a single record.

It had been the general experience in our unit that the discharge summary provided the information needed in practically every readmission. This experience, however, had not been formally tested until the present study. We have now confirmed that, in the sample of records tested, the summary alone was sufficient in about $95 \%$ of cases. With use of the criteria in our study the discharge summary may be further condensed to a few essential facts, representing one-fifth of the original summary. It appears that all these facts could be numerically coded for computer storage. Again we emphasise that this is not necessarily true of all specialties.

This study could be extended. A group of physicians and surgeons who had some experience with the method might take a list of discharge diagnoses in gastroenterology together with a list of operations performed and agree on a list of investigations that ought to be recorded for each diagnosis and operation. In this way a complete "investigation" file could be built up. It would be for experiment to determine whether non-medical staff could satisfactorily extract the essential information from notes to make the summarised record. After some years the method could be tested as outlined here on patients who were readmitted to see if it provided sufficient information. Such a study, in whatever specialty, might become the responsibility of a specialist society. 
If clinical medicine is to be formalised as a sequence of decisions, some education of clinicians in the newer way of thinking is necessary. At least one way would be to undertake the kind of study we have described, which forced us to analyse the process of decision making and give numerical estimates to some of the variables. Our experience was most educative and can be recommended.

WIC acknowledges with gratitude a grant from the Scottish Home and Health Department. We are grateful to Professor J P A McManus for help in the early stages of this study.

\section{References}

${ }^{1}$ Weed, L L, Medical Records, Medical Education and Patient Care. Cleveland, Ohio, Western Reserve University, 1969.

${ }^{2}$ Feinstein, A R, Annals of Internal Medicine, 1973, 78, 751.

${ }^{3}$ Lindley, D V, Making Decisions. London, Wiley Interscience, 1971.

4 Card, W I, and Good, I J, in Companion to Medical Studies, ed R Passmore and J S Robson, vol 3, p 60. Oxford, Blackwell Scientific, 1974.

5 Good, I J, and Card, W I, Mathematical Biosciences, 1971, 10, 157

6 Platt, R, Lancet, 1952, 2, 977.

7 Doll, R, Proceedings of the Royal Society of Medicine, 1968, 61, 709.

\title{
Controlled comparison of cimetidine and carbenoxolone sodium in gastric ulcer
}

\author{
S J LA BROOY, R H TAYLOR, R H HUNT, P L GOLDING, J M LAIDLAW, R G CHAPMAN, \\ R E POUNDER, S H VINCENT, D G COLIN-JONES, G J MILTON-THOMPSON, J J MISIEWICZ
}

British Medical fournal, 1979, 1, 1308-1309

\section{Summary and conclusions}

Fifty-four outpatients with endoscopically diagnosed benign gastric ulcer were allocated at random to treatment with either cimetidine $800 \mathrm{mg}$ daily for six weeks or carbenoxolone sodium $300 \mathrm{mg}$ daily for one week then $150 \mathrm{mg}$ daily for five weeks. Ulcers were reassessed by endoscopy at the end of the trial. The endoscopist was unaware of the treatment and did not take part in the clinical care of the patients.

Twenty-one of the 27 patients $(78 \%)$ given cimetidine and 14 of the $27(52 \%)$ given carbenoxolone had healed ulcers. Symptomatic response occurred earlier with cimetidine but was not significantly better. Unwanted effects were more common in the carbenoxolone group: 12 patients developed hypokalaemia, four of whom needed oral potassium supplements.

The results suggest that histamine $\mathrm{H}_{2}$-receptor blockade is at least as effective as carbenoxolone sodium for benign gastric ulcer and produces fewer side effects.

\footnotetext{
Department of Gastroenterology, Central Middlesex Hospital, London NW10 7NS

S J LA BROOY, $M B, M R C P$, research registrar

R H TAYLOR, MB, MRCP, research senior registrar

R E POUNDER, MRCP, DM, research senior registrar (now senior registrar,

St Thomas's Hospital, London)

J J MISIEWICZ, MB, FRCP, consultant physician

Department of Medicine and Gastroenterology, Royal Naval Hospital, Haslar, Hampshire

$S$ H VINCENT, $M B, B S$, registrar

R H HUNT, MB, MRCP, consultant physician

G J MILTON-THOMPSON, MD, FRCP, professor of naval medicine

Department of Gastroenterology, Queen Alexandra Hospital, Portsmouth

P L GOLDING, MB, MRCP, registrar

J M LAIDLAW, MB, MRCP, registrar

R G CHAPMAN, MB, MRCP, registrar

D G COLIN-JONES, MB, MRCP, consultant physician
}

\section{Introduction}

Controlled trials have suggested that carbenoxolone sodium accelerates the healing of gastric ulcer, but side effects limit the $\vec{\varphi}$ freedom with which it may be used, especially in the elderly. ${ }^{2}$ Despite this it has been generally accepted in the treatment of benign gastric ulcer. Early, uncontrolled studies showed that the $\mathrm{H}_{2}$-receptor antagonist cimetidine might be effective in benign gastric ulcer and free of unwanted side effects. ${ }^{3}$ In a later, controlled trial cimetidine was found to be significantly better than a placebo. ${ }^{4}$ We report the results of a controlled study comparing cimetidine $800 \mathrm{mg} /$ day for six weeks with the recommended dose of carbenoxolone sodium in the short-term treatment of benign gastric ulcer.

\section{Patients and methods}

On study day 0 a lesser-curve gastric ulcer was diagnosed endoscopically. Four quadrant biopsy specimens and brush-cytological smears were taken from each ulcer, and blood was obtained for routine haematological and biochemical safety screening. All medication was withdrawn except for a counted supply of unmarked antacid tablets $\mathrm{S}$ containing aluminium hydroxide $180 \mathrm{mg}$ and magnesium hydroxide $N$ $60 \mathrm{mg}$. Patients were instructed in the use of a diary card for the daily recording of daytime and night-time pain and consumption of antacid.

Patients with prepyloric, coexisting duodenal, or postoperative ulcers or whose gastric ulcer was less than $1 \mathrm{~cm}$ diameter (measured $N$ by comparison with an open biopsy forceps) were excluded from the $\underset{\omega}{N}$ study. Also excluded were patients aged 18 years or less, pregnant 0 and lactating women, and patients with hypertension or cardiovascular or renal disease. Those with histopathological or cytological evidence of malignancy or with abnormal laboratory results on the safety $\mathscr{\cap}$ screen were withdrawn.

Patients were seen again on day 7. Those fulfilling the criteria for the trial were allocated at random to treatment with either cimetidine $200 \mathrm{mg}$ four times daily after meals for six weeks or carbenoxolone $\mathcal{O}$ sodium $100 \mathrm{mg}$ thrice daily for one week then $50 \mathrm{mg}$ thrice daily for $\stackrel{\mathbb{Q}}{2}$ five weeks. All patients were seen weekly. At each visit diary cards and unused antacid and trial tablets were collected and a new supply issued. The frequency of pain and antacid consumption were recorded, 8 the patient weighed and examined, and blood obtained for routine haematological and biochemical studies.

Within three days after the end of the six-week course of treatment each patient underwent repeat endoscopy, the result being recorded as "ulcer healed" or "ulcer not healed." The same endoscopist performed both examinations. The endoscopist had no part in clinical management and remained unaware of the patients' treatment. 